\title{
Design and Development of a GSM-based Smart Home Automation System
}

\author{
Md. Selim Reza ${ }^{1}$, SM Mamun ${ }^{2}$, Monjur Mossadded Munna ${ }^{3}$ \\ ${ }^{1}$ (Lecturer, Department of Natural Sciences, BGMEA University of Fashion \& Technology, Bangladesh) \\ ${ }^{2}$ (Professor, Department of EEE, University of Dhaka, Bangladesh) \\ ${ }^{3}$ (Assistant Engineer, Essen Engineering Company Ltd., Bangladesh)
}

\begin{abstract}
:
Home Security Systems are very important features of modern residential and office setups. This system is used to keep home safe from harmful gases, fire and intruders. It must be affordable, reliable and effective. In this paper, a GSM based home security system has been designed and implemented. The system consists of a GSM Module, MQ-2 smoke detector, Ultrasonic sonar sensor, LM35 temperature sensor, Arduino Uno and relay module. The MQ-2 smoke detector is used to detect harmful gases i.e. $\mathrm{LPG}, \mathrm{CH}_{4}$, $\mathrm{CO}$, Alcohol, Smoke or Propane. Any of the harmful gases stated above is detected it will warn the owner by activating the buzzer and displaying a warning message on the LCD display. It will also send a SMS to the owner. Our system can also measure temperate and if the temperature is increased above a certain range it will compensate it by activating Air Conditioner through the relay module. The system will also send a SMS to the owner. It can also detect intruders using Ultrasonic sensor and warn us through buzzer, SMS and displaying message on the LCD. Our system is reliable as it has very fast response and cost effective and it has been developed using our own technologies.
\end{abstract}

Keywords: GSM Module; Arduino; SMS; Relay; MQ-2; HC-SR04; LM35

\section{Introduction:}

In this world of advanced technology home automation has become one of the quickest developing application-based technologies. The intension of developing home automation system has changed for the past decade due to the advancement of digital, vision and wireless technologies. Automated homes can simply be described as homes that are fully automated in terms of performing a predetermined task, providing feedback to the users and responding quickly and accordingly to situations [1][2]. It is comprised of many modules including temperature and lighting control, unauthorized access control, refreshment and entertainment control, emergency response and security monitoring modules which can be operated effectively both from near and far regions [3][4]. There has been a tremendous effort to the design and development of home automation or security system throughout the world. Tan, Lee and Soh (2002) proposed the development of an internetbased system which can monitor important process variables from a distributed control system (DCS). They proposed hardware and software design considerations using a commonly available web browser that could enable the user to access the process variables on the DCS remotely and effectively [5]. In their paper, Liang, Fu, and $\mathrm{Wu}$ (2002) proposed a multi-agent based software architecture of home automation system. Their architecture is composed of five main components namely space agents, function agents, personal preference agents, environment variables server and resource access right control kernel [6]. There are still some scopes of improvement to construct a more powerful home automation system both in the hardware and software aspects. We have made a big contribution in the promotion and reliability of home automation system. Among them, the task of providing automatic short text message (SMS) notification regarding any unusual situations is user friendly and more reliable than other versions of home automation system. We are proposing the development of a home automation system which can ensure the safety and security asset in a more reliable way and can meet technological advancement to automate life providing maximum protection. So the aim of this paper is to design and implement an up-todate, reliable, cost effective and user friendly home automation system using Arduino synchronized with GSM module to provide maximum possible security. 


\section{Design \& Development:}

Our system is made up of two modules namely hardware and software. Hardware provides required signals to the computer in digital from and software analysis the acquired signal to provide the desire output as shown in the block diagram (figure 1).To implement our desired system, we used Microcontroller, Arduino UNO, GSM module, Lighting System, Sensors (Temperature, Smoke Detector \& Ultrasonic HC-SR04), LCD, Relay, etc. in hardware module. As software we used the Arduino IDE which is used to program the Arduinos. This software is written in JAVA. And the language it uses to program the $\mathrm{MCU}$ is like $\mathrm{C} / \mathrm{C}++$. We also used Proteus v7.7 for simulating the circuit.

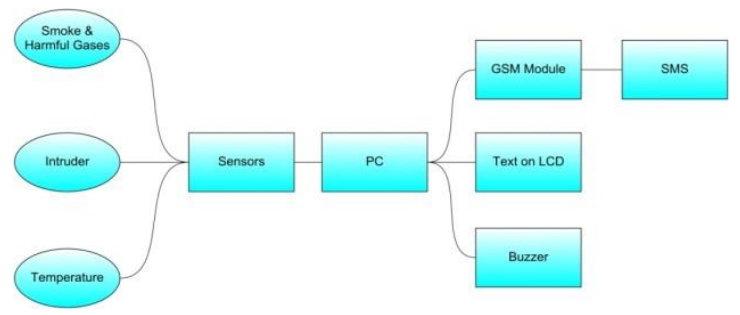

Figure 1: Block diagram of our system

We used MQ-2 gas sensor to detect smoke. It is a semiconductor type active low gas sensor used to detect combustible gas and smoke. The sensitive material of the sensor is tin dioxide $\left(\mathrm{SnO}_{2}\right)$. When it is exposed to combustible gas then at the surface of tin dioxide, oxidation reaction between the gas and adsorbed oxygen occurs. As a result, the density of adsorbed oxygen on the tin dioxide surface decreases. Hence the height of the potential barrier are reduced and electrons easily flow through them making the sensor resistance to decrease [7][8].The output voltage is than feed to a comparator (LM393) which gives digital output as shown in figure 2 .

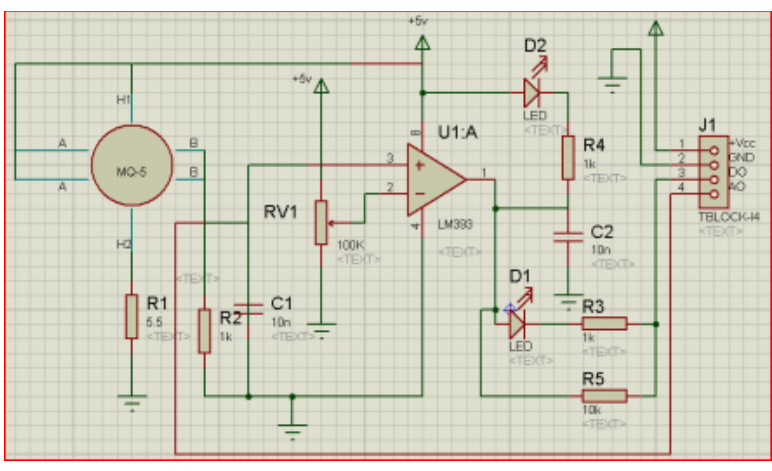

Figure 2: Working principle of MQ-2 sensor

To detect intruders we used ultrasonic sonar sensor (HC-SR04). It measures distance by sending out a sound wave with a specific frequency and listening for that particular wave to bounce back as shown in figure 3. By recording the elapsed time between the sound wave being sent and bounced back, it is possible to calculate the distance between the sonar sensor and the object. Since it is known that sound wave travels through air with a speed of $344 \mathrm{~m} / \mathrm{s}(1129 \mathrm{ft} / \mathrm{s})$ we can take the time for the sound wave

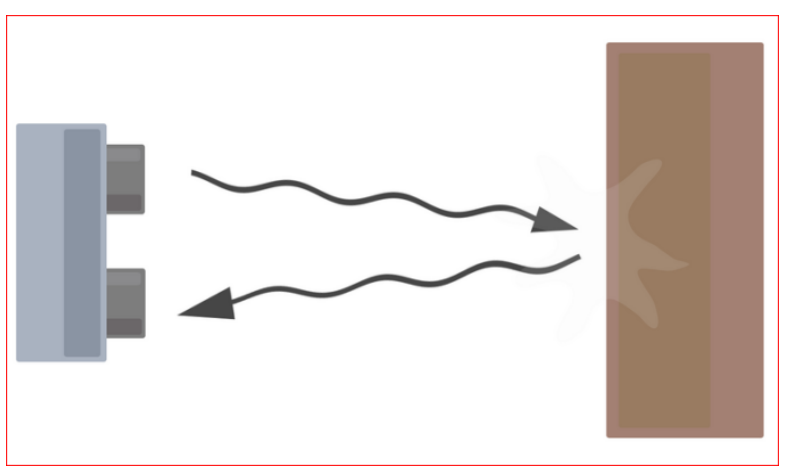

Figure 3: Diagram of the basic ultrasonic sensor operation

to return and multiply it by 344 meters (or 1129 feet) to find the total round-trip (round-trip means that the sound wave travels two times the distance to the object before it was detected by the sensor including the trip from the sonar sensor to the object and the trip from the object to the sensor) distance of the sound wave. To find the distance to the object we need to divide the round-trip distance by two [9][10].

To monitor the temperature we used LM35 IC which is a precision temperature sensor with its output proportional to ${ }^{0} \mathrm{C}$. The sensor circuitry is sealed and 
therefore it is not subjected to oxidation \& other processes and can measure temperature more accurately than a thermistor. The sensor's operating temperature ranges from $-55^{\circ} \mathrm{C}$ to $150^{\circ} \mathrm{C}$. Its output voltage varies by $10 \mathrm{mV}$ in response to every ${ }^{0} \mathrm{C}$ rise or fall in ambient temperature [11].

We used GSM module SIMcom SIM900A which is an ultra-compact and reliable wireless module works on frequencies $900 / 1800 \mathrm{MHz}$ to send SMS. If anything unusual happens the system will send SMS to the owner using this module [12].The complete developed system is shown in figure 4.

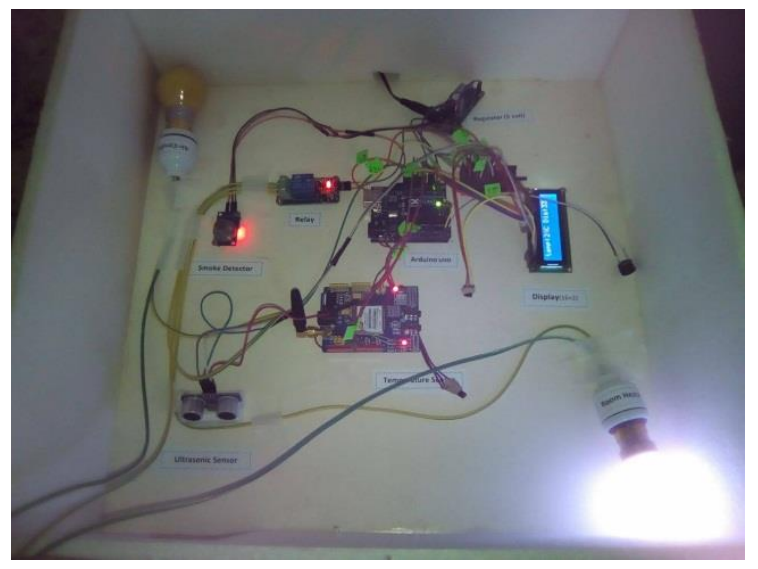

Figure 4: Our complete developed system.

\section{Result \& Analysis:}

\section{Testing:}

Our developed prototype system has been tested with the mobile network. It was found to have very fast response time and location independent as user can get alerts about any unusual occurrences from anywhere through the GSM technology.

\section{Temperature Sensor:}

The system will measure temperature and if the temperature is increased above a certain predefined range $\left(45^{\circ} \mathrm{C}\right.$ in our case $)$ it will warn us by activating buzzer. A warning message will be shown on LCD. The unusual condition will be compensated by turning
ON the Air Conditioner through activation of relay module. The system will also send SMS to the owner using GSM module. The following flow chart shows the working mechanism of temperature monitoring and detection part of our system.

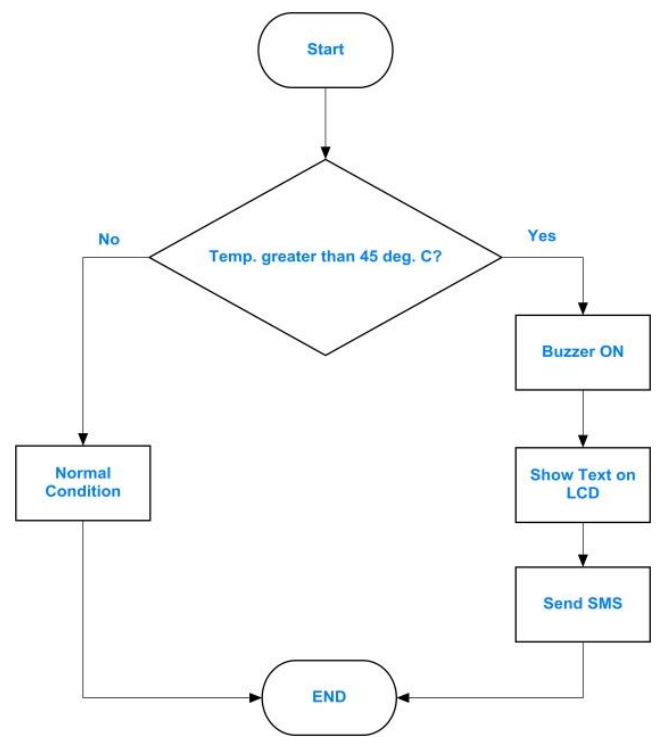

Figure 5: Flow chart of temperature detection system.

\section{Smoke Sensor:}

The MQ-2 smoke detector is used to detect harmful gases i.e. $\mathrm{LPG}, \mathrm{CH}_{4}, \mathrm{CO}$, Alcohol, Smoke or Propane. Any of the harmful gases stated above is detected it will warn by activating the buzzer, displaying a warning message on the LCD display and sending a SMS to the owner. The following flow chart shows the working mechanism of the smoke sensor in our system. 


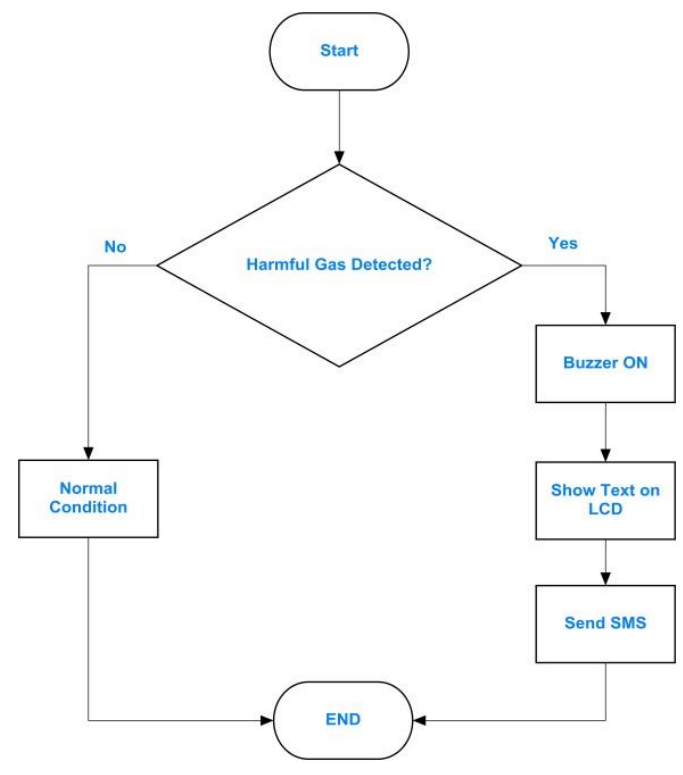

Figure 6: Flow chart of harmful gas detection section.

\section{Ultrasonic Sensor:}

It can detect intruders using Ultrasonic sensor and warn us through buzzer, SMS and displaying message on the LCD. Our system is reliable as it has very fast response and cost effective as it has been developed using our own technologies.

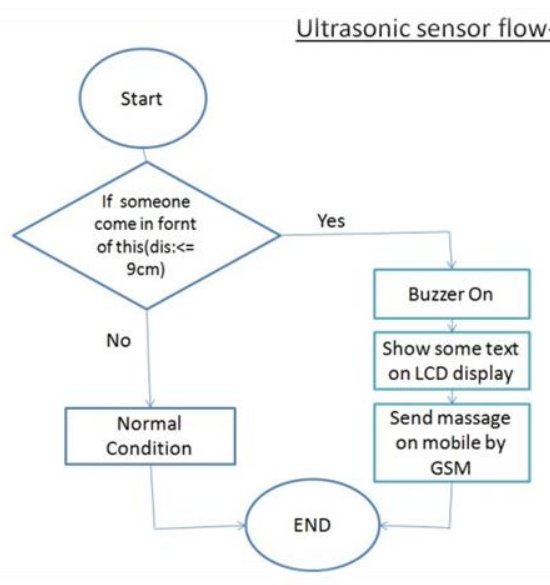

Figure 7: Flow chart of intruder's detection mechanism.

We have tested our system and found its response as fast and accurate. The table blow shows our test results under difference circumstances. It shows that the detection range is small as we used low cost sensors.

\begin{tabular}{|l|l|l|l|l|l|}
\hline \multicolumn{2}{|c|}{$\begin{array}{r}\text { Temperature } \\
\text { Sensor }\end{array}$} & \multicolumn{2}{c|}{ Smoke Sensor } & \multicolumn{2}{|c|}{$\begin{array}{l}\text { Ultrasonic } \\
\text { Sensor }\end{array}$} \\
\hline $\begin{array}{l}\text { Distanc } \\
\text { e }\end{array}$ & $\begin{array}{l}\text { Respons } \\
\text { e }\end{array}$ & $\begin{array}{l}\text { Distanc } \\
\text { e }\end{array}$ & $\begin{array}{l}\text { Respons } \\
\text { e }\end{array}$ & $\begin{array}{l}\text { Distanc } \\
\text { e }\end{array}$ & $\begin{array}{l}\text { Respons } \\
\text { e }\end{array}$ \\
\hline $10 \mathrm{~cm}$ & $\begin{array}{l}\text { Yes } \\
\text { (fast) }\end{array}$ & $10 \mathrm{~cm}$ & $\begin{array}{l}\text { Yes } \\
\text { (fast) }\end{array}$ & $10 \mathrm{~cm}$ & $\begin{array}{l}\text { Yes } \\
\text { (fast) }\end{array}$ \\
\hline $20 \mathrm{~cm}$ & $\begin{array}{l}\text { Yes } \\
\text { (fast) }\end{array}$ & $15 \mathrm{~cm}$ & $\begin{array}{l}\text { Yes } \\
\text { (fast) }\end{array}$ & $15 \mathrm{~cm}$ & $\begin{array}{l}\text { Yes } \\
\text { (fast) }\end{array}$ \\
\hline $100 \mathrm{~cm}$ & No & $100 \mathrm{~cm}$ & No & $100 \mathrm{~cm}$ & $\begin{array}{l}\text { Yes } \\
\text { (slow) }\end{array}$ \\
\hline $200 \mathrm{~cm}$ & No & $200 \mathrm{~cm}$ & No & $200 \mathrm{~cm}$ & No \\
\hline
\end{tabular}

Table: Observation of test result of different units under certain circumstances

\section{Conclusion:}

A GSM based smart home automation system has been designed and developed. It can detect and warn about any types of unauthorized access by activating buzzer and sending SMS to predefined users. It can also monitor temperature and compensate increase or decrease in temperature above or below a predefined range automatically by activating the refrigerator. Besides the system can detect combustible gases (i.e. LPG) and warn us by activating the buzzer and sending SMS to the predefined users. Our designed system is reliable, effective and user friendly. Some of the key features of the system are mentioned below:

i) It can be controlled by mobile phone through GSM.

ii) It can be controlled within a reasonable range like outside of home when room is locked.

iii) It is cheap, easily maintainable and user friendly.

\section{Future Works:}

We have designed a prototype system with low cost\& small detection ranged sensors. If we replace those with industrial sensors than the system can easily and effectively be implemented in homes. We can add some more sensors i.e. humidity sensor, motion sensor, etc. to make it a perfect one. More ever we can 
integrate sensors in multiple PCB to cover a full premise. We can also use wireless module to increase our system's efficiency.

\section{References:}

[1] Baris Yuksekkaya, A. Alper Kayalar, M. Bilgehan Tosun, M. Kaan Ozcan, and Ali Ziya Alkar, “A GSM, Internet and Speech Controlled Wireless Internet Home Automation System", IEEE Transactions on Consumer Electronics, Vol. 52, No. 3, AUGUST 2006

[2] Kwang Yeol Lee \& Jae Weon Choi, "RemoteControlled Home Automation System via Bluetooth Home Network" in SICE Annual Conference in Fukui, 2003, Vol. 3, pp. 2824-2829

[3] Saisakul Chernbumroong, Anthony S. Atkins, and Hongnian $\mathrm{Yu}$, "Perception of Smart Home Technologies to Assist Elderly People" 4th International conference on software, Knowledge information manage and applications (SKIMA 2010)

[4] Sandeep Kumar \& Mohammed A Qadeer, "Universal Digital Device Automation and Control", in $2^{\text {nd }}$ IEEE International Conference on Computer Science and Information Technology, 2009, pp. 490494

[5] KokKiong Tan, Tong Heng Lee and Chai Yee Soh, "Internet-based monitoring of distributed control systems-An undergraduate experiment," in IEEE Transactions on Education, vol. 45, no. 2, pp. 128-134, May 2002

[6] Neng-Shiang Liang, Li -Chen Fu, Chao-Lin Wu," An integrated, flexible, and Internet- based control architecture for home automation system in the Internet Era," in procedings of the 2002 IEEE International Conference on Robotics \& Automation, Washington DC, May 2002

[7] Md. Selim Reza, S.M. Mamun, Md. Abdullah Al Mamun, Md. Atiar Rahman,"PC Based Wireless Monitoring and Control of Fire Detection and Extinguishing System" International Journal on Engineering Applications (IREA), Praise Worthy Prize (USA), Vol. 3, May-2015

[8] Technical data of MQ-2 sensor (https://www.pololu.com/file/0J309/MQ2.pdf)
[9] M. Asadullah and K. Ullah, "Smart home automation system using Bluetooth technology," 2017 International Conference on Innovations in Electrical Engineering and Computational Technologies (ICIEECT), Karachi, 2017, pp. 1-6.

[10] The Ultrasonic sonar sensor (http://education.rec.ri.cmu.edu/content/electronics/b oe/ultrasonic_sensor/1.html)

[11] Workshop on LabVIEW, Institute of Information Technology (IIT), University of Dhaka, 2018

[12]SIM900A wireless module (http://www.instructables.com/id/GSM-SIM900AWith-Arduino/) 